OUR ASTRONOMICAL COLUMN.

The Red Spot on Jupiter.-This object exhibited a slackening motion during the years from 1878 to I900. It then became decidedly accelerated, so that the rotation period, which in 1899 and 1900 was $9 \mathrm{~h} .55 \mathrm{~m}$. $4 \mathrm{I} \cdot 7 \mathrm{~s}$. , decreased in $190 \mathrm{I}$ to $9 \mathrm{~h} .55 \mathrm{~m}$. $40 \cdot 6 \mathrm{~s}$., and in 1902 to $9 \mathrm{~h} .55 \mathrm{~m}$. 39.0s. In 1903 the spot again became retarded, and the rotation period increased to $9 \mathrm{~h} .55 \mathrm{~m} .4 \mathrm{I} \cdot \mathrm{os}$. This retardation has now in turn given way to another acceleration of speed. In January last the longitude of the spot was $35^{\circ}$, whereas at the present time it is only $30^{\circ}$, so that the rotation period during the first six months of 1904 has been about $9 \mathrm{~h} .55 \mathrm{~m} .39 \cdot 5 \mathrm{~s}$. It is difficult to explain these curious oscillations in velocity. Some extensive disturbances have, however, affected the south temperate region of the planet in recent years, and a large dusky patch has been visible since $190 \mathrm{r}$ rotating with a rate of $9 \mathrm{~h} .55 \mathrm{~m}$. $18.7 \mathrm{~s}$., or about 22 seconds less than that of the red spot. The motion of the latter may possibly have been affected by disturbances occurring in the same latitude, but this can only be fully determined by further observations. In the meantime, both the red spot and the south temperate spot are being attentively watched as to their motions and appearances. The two objects were in conjunction in July, I9O2, and June, 1904, and in the spring of 1906 the event will be repeated if the south temperate spot should remain visible until that time. As to the red spot and its surroundings, they appear to form features of remarkable permanency, and are likely to continue perceptible for an indefinite period.

Variable Radial Velocity of $\alpha$ Andromede and Four OTHER Stars.-Whilst engaged in line-of-sight work with the Lowell spectrograph Mr. V. M. Slipher discovered the variable radial velocities of $\alpha$ Andromedæ, $\alpha$ Libræ, $\sigma$ Scorpii, X Sagittarii, and $\epsilon$ Capricorni.

The velocities of $\alpha$ Andromedæ were obtained from measurements of the $\mathrm{H} \gamma$ and 448I magnesium lines, the helium $447^{2}$ line also being measurable. They range from +20 (February II) to $-45 \mathrm{~km}$. (March 4), but are uncertain to a few kilometres. The observations indicate a period of about 100 days and a very eccentric orbit. range between $-60 \mathrm{~km}$. on May 24 and $+20 \mathrm{~km}$. on July 6 , and suggest that both components are bright. The velocities of $\sigma$ Scorpii range between +25 (June 25 ) and $-25 \mathrm{~km}$. (June I8). Only two plates were measured for X Sagittarii, which is a visual variable having a period of seven days, and these gave $+\mathrm{r}$ and $-22 \mathrm{~km}$. on June 19 and $22 \mathrm{re}-$ spectively. A range of from $-45 \mathrm{~km}$. (September 7 , 1903) to $+6 \mathrm{~km}$. (July 6) was obtained for the radial velocity of $\epsilon$ Capricorni (Lowell Observatory Bulletin, No. Ir).

Various Classes of Silicium Lines and their OccurRENCE IN Stellar Spectra.--In a communication to l'Académie des Sciences (Paris), M. de Gramont describes some results he has obtained during a series of experiments on the effects of various amounts of self-induction in the spark spectrum of silicium. His observations led him to form two main classes of silicium lines:(I) those which are not affected or are strengthened by self-induction amounting to 0.03 henry; (2) those of which the intensities are reduced by self-induction and which disappear entirely with 0.006 henry.

He further divides them into eight groups $(\alpha-\eta)$, and, in a table showing their individual characteristics in the spark and in various stellar spectra, he shows their connections with the four temperature groups (silicium i.-iv.) named by Sir Norman Lockyer in his temperature classification of the stars. From this table he draws the following conclusions:-(x) Only the spectra of the first class, i.e. hydrogen and helium stars, show the lines which disappear under the action of self-induction, those of helium, e.g. the Orion stars, exhibiting as strong lines those which are first to disappear (Lockyer's silicium iii.), whilst the hydrogen stars, e.g. Sirius, present the lines which are the last to disappear (silicium ii.). Stellar spectra of the second class (solar type) and the "flash spectrum" contain the lines which appear in both arc and spark, and resist self-induction, e.g. $\lambda$ 3905.7 (silicium i.). As the lines belonging to Sir Norman Lockyer's group iv. are near oxygen and nitrogen lines, and always disappeared from the spectra with the air lines, and as oxygen and nitrogen have been shown No. I 8 I 4 , VOL. 70] to exist in the absorbing atmospheres of the stars the spectra of which show this group (e.g. $\beta$ Crucis), M. Gramon suggests that these lines are attributable to air (Comptes rendus, No. 3, vol. cxxix.)

Line of Sight Constants for some Orion Type Stars. -In No. 3, vol. xix., of the Astrophysical Journal, Miss E. E. Dobbin gives a list of line-of-sight constants for $\mathrm{I}_{22}$ stars of the Orion type, computed for the reduction of the Bruce spectrograph observations.

Dr. Schlesinger's formulæe, as employed in his "Line-of Sight Constants for the Principal Stars," were used, and the name, magnitude, position, and constants are given for each star. The longitude is given for 1900 , and therefore requires the $50^{\prime \prime}$ precession correction for each year since then.

The Tails of Borrelly's Comet (I903) and LigiltPRESSURE.-Mr. S. A. Mitchell, of Columbia University, has calculated the value of the repulsive force due to lightpressure which acted on the several tails of Borrelly's comet Using the values for the angle between the radius vector of the comet's path and the tail, as determined by Prof. Albrecht, he found somewhat discordant values for the principal tail, which gave, in the mean, the value for the light pressure as 18.47 times gravity. For the secondary tail the values were much more consistent, and gave a mean of $\mathrm{I} .824$ times gravity; the last four lines given in this table, which were derived from measures of the angle on August $13,14,15$, and 18 , give a mean for the repulsive force of 1.460 times gravity, and therefore appear to indicate the existence of a third tail, which the photographs obtained or August 12 and 15 corroborated.

In a second table Mr. Mitchell compares the values of the angles between the tails and the radii vectores as obtained (I) by calculation from the repulsive forces given above, (2) by direct measurement. The results agree fairly well considering the uncertainty of the measures of such illdefined objects as the tails. The differences between the observed and calculated values for the principal tail as the comet approached the sun indicate the presence of some other repulsive force in addition to that caused by light pressure, and $\mathrm{Mr}$. Mitchell believes that part of this, at least; is real. The size of the particles forming each of the three tails, as determined from the above repulsive forces, was $0 \cdot \mathrm{I} \mu, \mu$, and $\mathrm{I} \cdot 33 \mu$ respectively (Astrophysical Journal, No. I, vol. xx.).

SuRvey OF INDIA, 190I-2.--A volume of " Extracts from Narrative Reports of the Survey of India, rgoI-2," published at Calcutta (1904), contains accounts of the work done by several parties of surveyors in connection with the triangulation of Upper Burma, latitude operations, the magnetic survey of India, tidal and levelling operations, and the topography of Upper Burma, Sind, and the Punjab.

During the latitude operations some puzzling anomalies were discovered between the observed and calculated values, ihe difference $\mathrm{O}-\mathrm{C}$ preserving its positive character to a point much further north than might be expected.

The latitude results obtained, using stars from Newcomb's catalogue and from the Greenwich ten-year catalogue for 1880 , show the same probable errors, but there is a noteworthy consistency of sign and amount (about $\left.+0 \cdot 3^{\prime \prime}\right)$ in the value Newcomb-Greenwich.

An interesting account of the practical details of the magnetic survey, and of the instrumental equipments at Dehra Dun, Kodaikanal, Calcutta, and Rangoon are given in part iii., where the principles of several new and modified instruments are also fully described.

\section{THE BRITISH MEDICAL ASSOCIATION IN OXFORD.}

THE seventy-second annual meeting of the British Medical Association, which was held in Oxford last week (July 26 to 29), was beyond question one of the most successful meetings in the memory of members of the association, as it was in point of numbers much the largest yet recorded.

It was remarkable also for the persistence and enthusiasm with which, in spite of all the counter-attractions of that ancient and glorious seat of learning, and of the diversions, entertainments, and receptions arranged both by the 